\title{
Carcinoid Tumor in a Mature Thymic Teratoma: A Rare Type of Extra Cardiac Mass
}

\author{
Gregoriana Zanini, ${ }^{1}$ Mariella Chiudinelli, ${ }^{2}$ Luisa Bercich,, \\ Alessandra Pelati, ${ }^{1}$ Monica Bortolotti, ${ }^{1}$ and Gian Franco Pasini ${ }^{1}$ \\ ${ }^{1}$ U.O.C. Cardiologia, Ospedale la Memoria di Gavardo, Via Gosa n. 74, \\ 25085 Gavardo, Italy \\ ${ }^{2}$ Servizio di 1 Anatomia Patologica, Spedali Civili di Brescia (Brescia), Italy
}

Correspondence should be addressed to Gregoriana Zanini, gregoriana77@libero.it

Received 6 August 2012; Accepted 12 September 2012

Academic Editors: M. Sindel and G. L. Tripepi

Copyright ( $) 2012$ Gregoriana Zanini et al. This is an open access article distributed under the Creative Commons Attribution License, which permits unrestricted use, distribution, and reproduction in any medium, provided the original work is properly cited.

We describe the case of a young female affected by a thymus teratoma coexisting with carcinoid tumor.

\section{Introduction}

Malignant transformation of mature cystic teratoma is a rare complication occurring in approximately $1-3 \%$ of patients who have mature cystic teratoma. While any of the constituent tissues of a teratoma has the potential to undergo malignant transformation, squamous cell carcinoma is the most commonly associated malignancy [1]. The majority of mediastinal teratomas are mature teratomas that are histologically well defined and benign. Also mature teratomas do have the potential to undergo malignant transformation into a variety of malignancy (adenocarcinoma, rhabdomyosarcoma, leukemia) [1]. Thymic carcinoid is a malignant tumor: metastasis is common and the treatment is complete surgical resection. The prognosis of these tumors, when metastasis occur, are poor but difficult to assess because of little report in literature. Some authors described in kidneys a better prognosis for carcinoid tumor arising within teratomatous lesion compared to those arising within normal kidneys [2]. Thymus carcinoid tumors are rare and frequently associated with serotonin and neurotensin secretion. Thymus teratoma rarely presents together with carcinoid tumor. To the best of our knowledge, there has been only a report of thymus teratoma coexisting with carcinoid tumor [3].

\section{Case}

We present a rare case of typical carcinoid tumor arising within mature cystic teratoma of thymus in a 38-year-old female. Chest X-ray, done for incessancy cough, accidentally found an enlargement of the anterior mediastinum (Figure 1). Further ECG (Figure 2), transthoracic echocardiography, and then transoesophageal one (Figure 3) revealed an ovalar extracardiac mass immediately anterior to the first tract of the pulmonary artery and anterior to the left atrium without compression effect. She also underwent a thoracic medium contrast computerized axial tomography (Figure 4) that confirmed the encapsulated dishomogeneous expansive anterior mediastinal lesion of diameter $5.0 \times 7.5 \mathrm{~cm}$, with some calcification. She underwent to a surgical treatment with total thymectomy by left axillary thoracotomy saving muscles. Macroscopically the single chambered cystic lesion of about $8 \times 6 \times 3.7 \mathrm{~cm}$ was encapsulated and multiloculated with brown smooth thick wall filled with yellow-tan material. Inside the wall, there was a $1-1.5 \mathrm{~cm}$ nodular white area with calcification. Microscopically, the tumor showed coexistent mature cystic teratoma and moderately differentiated carcinoid tumor (Figures 5 and 6). The teratomatous cysts and carcinoid 


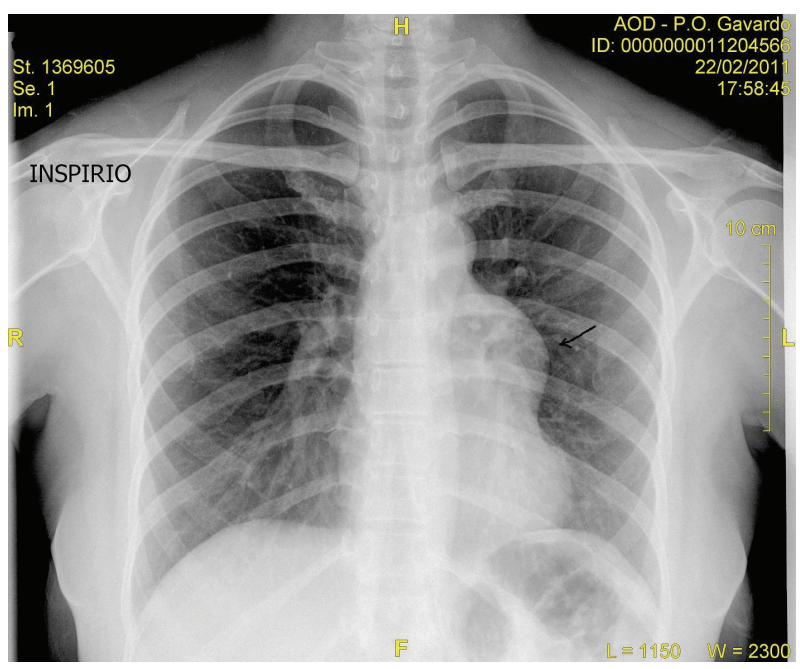

FIGURE 1: Chest X-ray in P-A: opacity mass of about $4.5 \mathrm{~cm}$ skull-caudal extension in left lateral anterior mediastinum.

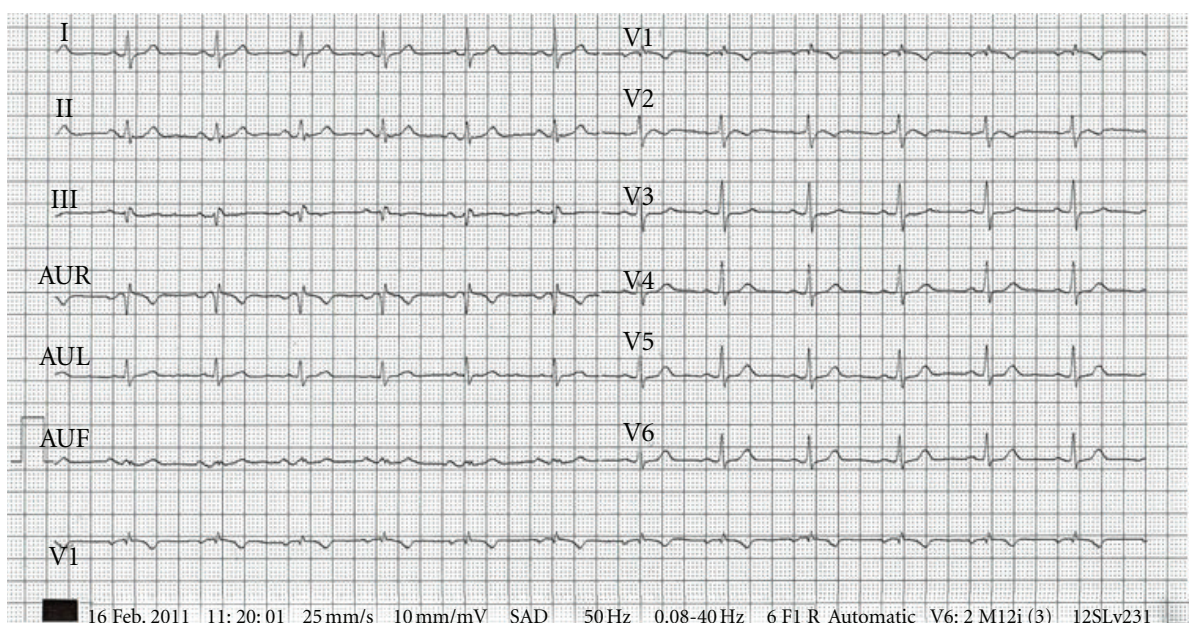

Figure 2: Electrocardiogram is normal.

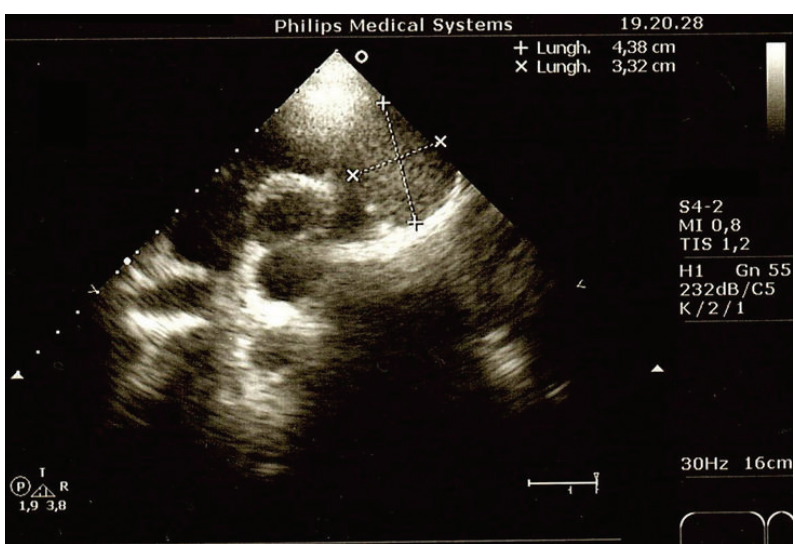

FIGURE 3: Transthoracic echocardiography with an ovalar iperecogenous mass of about $3.4 \times 4.3 \mathrm{~cm}$ near the left side of the pulmonary trunk. 


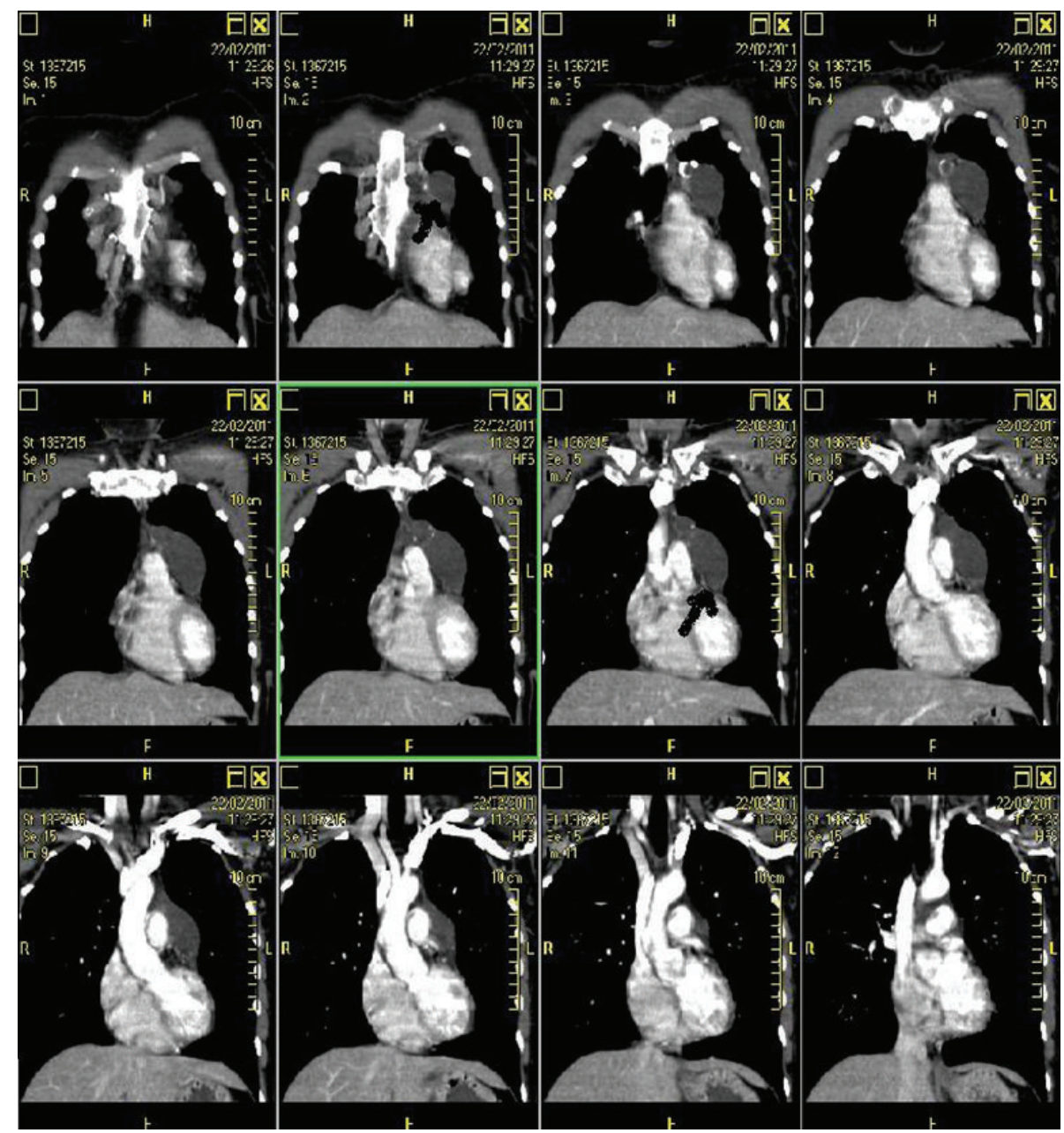

FIGURE 4: Thoracic medium contrast computerized axial tomography: encapsulated dishomogeneous expansive anterior mediastinal lesion of diameter $5.0 \times 7.5 \mathrm{~cm}$, with some calcification.

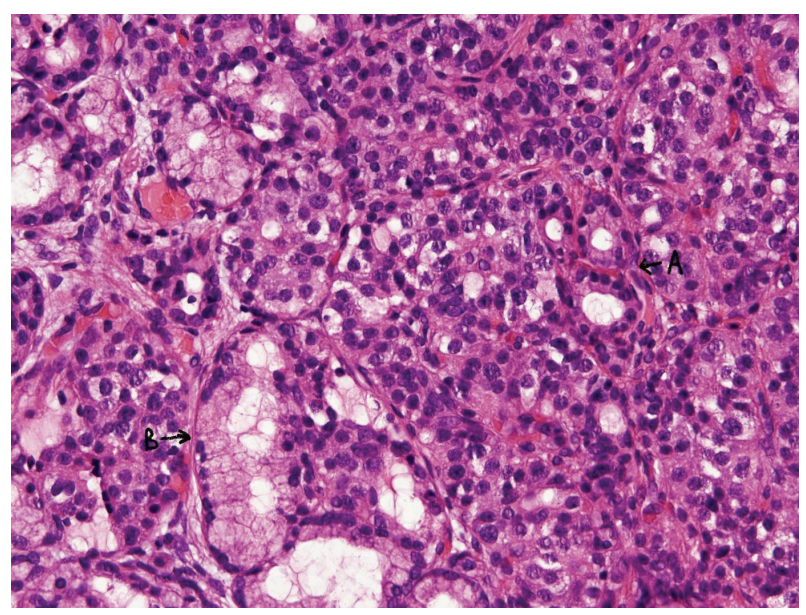

FIGURE 5: Microscopically (hematoxylin/eosin and cromogranina immunohistochemical staining): coexistent mature cystic teratoma (A) and moderately differentiated carcinoid tumor (B). 


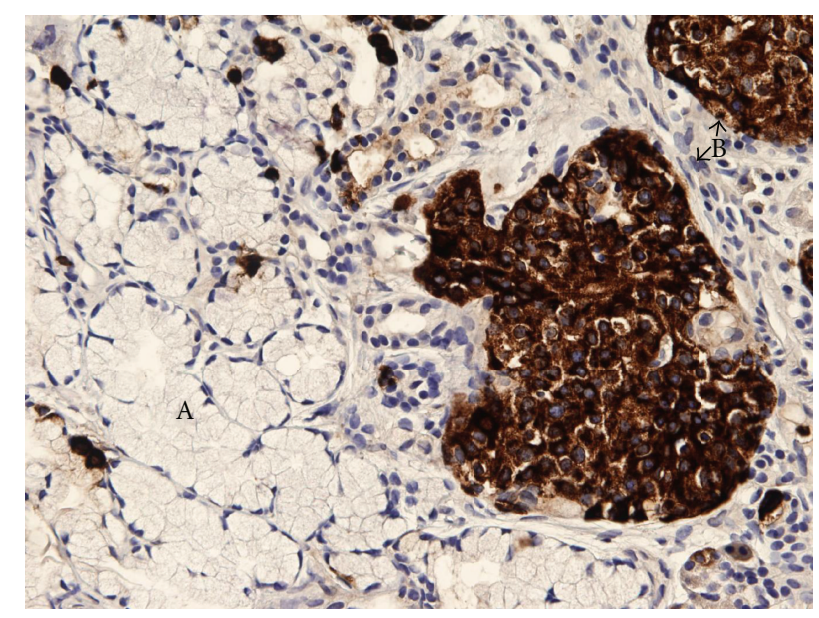

FIGURE 6: Microscopically (hematoxylin/eosin and cromogranina immunohistochemical staining): coexistent mature cystic teratoma (A) and moderately differentiated carcinoid tumor (B).

tumor showed strong staining for pankeratin, chromogranin and synaptophysin and insulin; negative for PTH, TTF1, gastrin, glucagon.

\section{Discussion and Conclusion}

This very rare case of extra cardiac mass emphasizes the need for thorough sectioning and entire submission for histologic evaluation of all the mass in particular of mature cystic teratomas, in order to avoid missing multiple additional histogenetically distinct neoplasms. The treatment for earlystage carcinoid tumors is surgery alone and excellent outcomes can be expected in these cases [4].

\section{References}

[1] B. V. Duwe, D. H. Sterman, and A. I. Musani, "Tumors of the mediastinum," Chest, vol. 128, no. 4, pp. 2893-2909, 2005.

[2] H. B. Armah, A. V. Parwani, and A. M. Perepletchikov, "Synchronous primary carcinoid tumor and primary adenocarcinoma arising within mature cystic teratoma of horseshoe kidney: a unique case report and review of the literature," Diagnostic Pathology, vol. 4, no. 1, article 17, 2009.

[3] B. S. Serezhin and M. I. Stepanov, "A carcinoid tumor in a mature thymic teratoma," Arkhiv Patologii, vol. 52, no. 7, pp. 59-62, 1990.

[4] S. B. Kale, D. S. Saksena, S. D. Kole, and Y. C. Agnihotri, "Carcinoid tumors of the thymus," Asian Cardiovascular \& Thoracic Annals, vol. 14, no. 2, pp. e30-e32, 2006. 


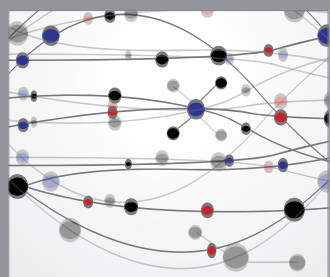

The Scientific World Journal
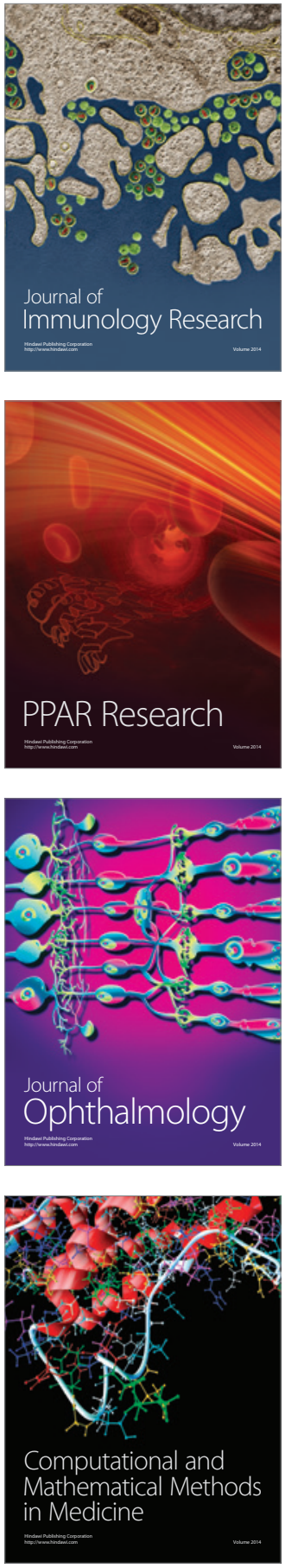

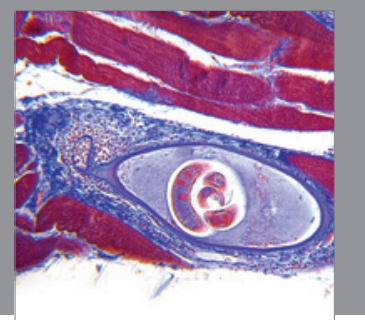

Gastroenterology

Research and Practice
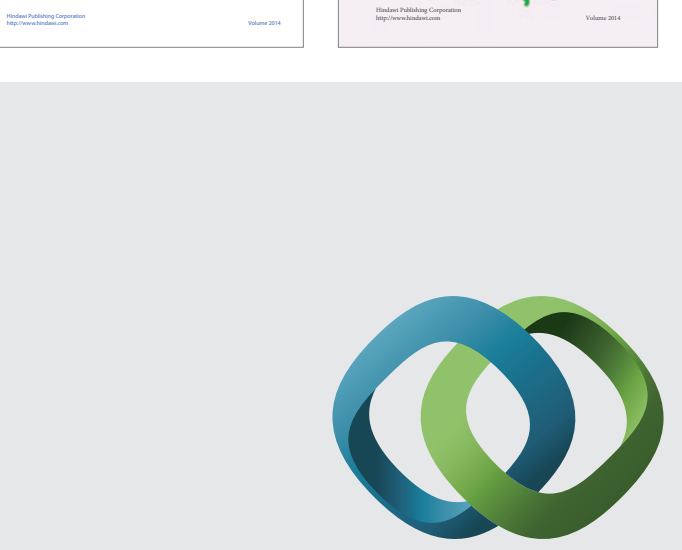

\section{Hindawi}

Submit your manuscripts at

http://www.hindawi.com
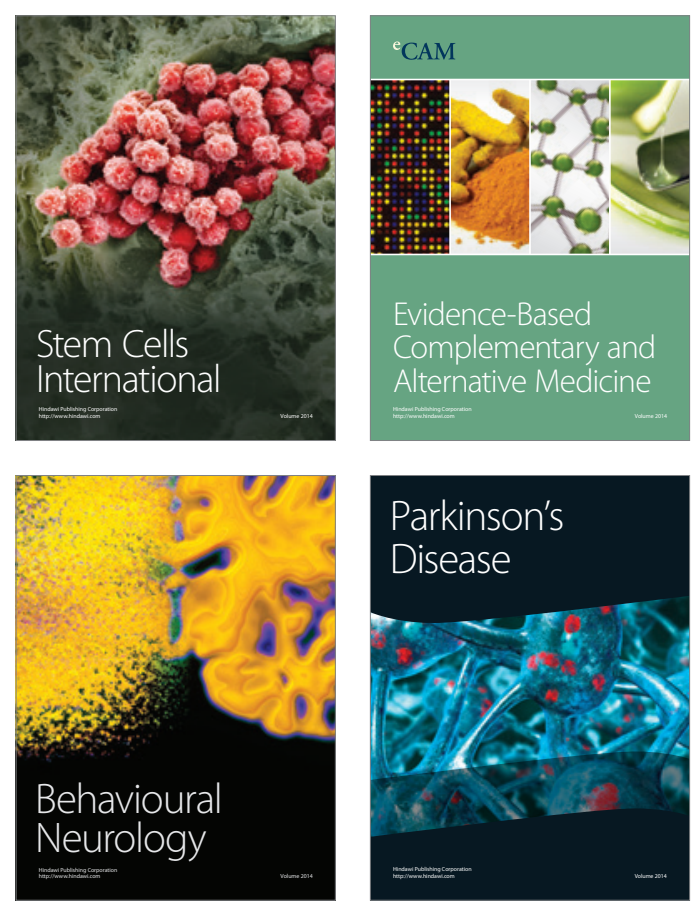

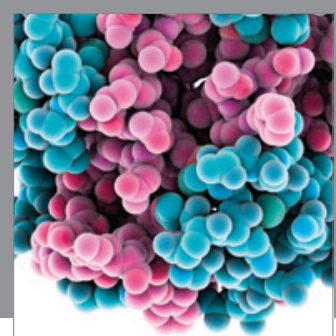

Journal of
Diabetes Research

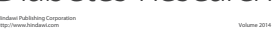

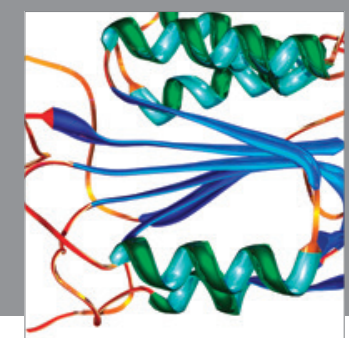

Disease Markers
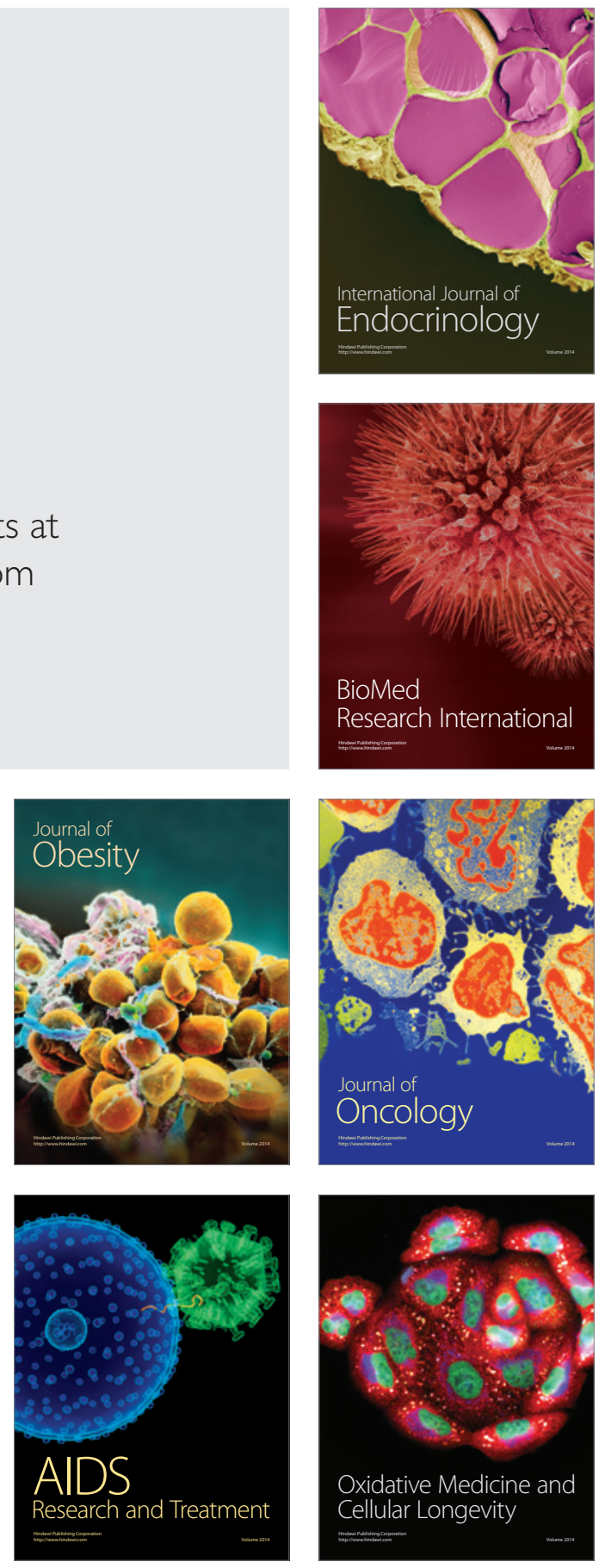